Vancomycin-associated acute kidney injury in critically-ill adolescent and young adult patients

\author{
William Blake Hays, Pharm.D. \\ Department of Pharmacy \\ Indiana University Health Methodist Hospital \\ 1701 N. Senate Blvd \\ Indianapolis, IN. 46202 \\ Phone: 317-963-0038 \\ whays@iuhealth.org \\ Emma Tillman, Pharm.D., Ph.D. \\ Department of Pharmacy \\ Indiana University Health Riley Hospital for Children \\ 705 Riley Hospital Drive \\ Indianapolis, IN. 46202 \\ Phone: $317-944-5000$ \\ etillman@iuhealth.org \\ Presented as research in progress at: \\ - Great Lakes Pharmacy Residency Conference 2017 \\ - Pediatric Pharmacy Advisory Group Annual Meeting 2017
}

Authors have no conflicts of interest to report

Word Counts:

Abstract: 250

Main Text: 1524

References: 24

Key Words: Vancomycin, Nephrotoxicity, Critical Care, Adolescent Medicine, Pharmacokinetics

This is the author's manuscript of the article published in final edited form as:

Hays, W. B., \& Tillman, E. (2019). Vancomycin-Associated Acute Kidney Injury in Critically III Adolescent and Young Adult Patients. Journal of Pharmacy Practice, 897190019829652. https://doi.org/10.1177/0897190019829652 


\title{
Vancomycin-associated acute kidney injury in critically-ill adolescent and young adult patients
}

\begin{abstract}
Background: Risk factors for the development of vancomycin-associated acute kidney injury have been evaluated in both pediatric and adult populations; however, no previous studies exist evaluating this in the critically-ill adolescent and young adult patients. Objective: Identify the incidence of acute kidney injury (AKI) and examine risk factors for the development of AKI in critically-ill adolescents and young adults on vancomycin. Methods: This retrospective review evaluated the incidence of AKI in patients 15-25 years of age who received vancomycin, while admitted to an intensive care unit. AKI in this population was defined as an increase in serum creatinine by $0.5 \mathrm{mg} / \mathrm{dL}$ or $50 \%$ from baseline. Patients who developed AKI were evaluated for specific risk factors compared to those who did not develop AKI. Results: A total of 50 patients ( 20 developed $\mathrm{AKI}$ ) were included in the study. There was no difference in vancomycin daily dose or duration of vancomycin therapy. Highest vancomycin trough $(31.15 \mathrm{mg} / \mathrm{dL}$ vs $12.5 \mathrm{mg} / \mathrm{dL}, \mathrm{P}=0.006)$, percentage of patients with concurrent nephrotoxic medication ( $95 \%$ vs $60 \%, P=0.012$ ) and concurrent vasopressor ( $55 \%$ vs $23 \%, P=0.029$ ) were higher in those who developed AKI. Percentage of patients who underwent a procedure while on vancomycin ( $35 \%$ vs $6.7 \%, \mathrm{P}=0.021$ ) was also higher within the $\mathrm{AKI}$ group. Conclusions: Vancomycin-associated AKI occurred in $40 \%$ of critically-ill adolescent and young adult patients. These patients may be more likely to develop vancomycin-associated AKI if they had undergone a procedure, as well as in the presence of high vancomycin trough levels, concurrent nephrotoxic agents, and concurrent vasopressor therapy.
\end{abstract}




\section{Background}

Vancomycin is a glycopeptide antibiotic that is commonly used for the empiric and targeted treatment of gram positive infections in critically ill patients. ${ }^{1-3}$ Achieving adequate treatment of these gram-positive infections, while avoiding adverse effects from vancomycin is a challenge that clinicians have been facing for many years. Vancomycin-associated acute kidney injury was initially attributed to impurities in the manufacturing of the product. ${ }^{4}$ It is now known that vancomycin directly produces renal tubular damage through oxidative stress, resulting in AKI. ${ }^{5,6}$ Factors such as concurrent use of other nephrotoxic agents, blood urea nitrogen (BUN) to serum creatinine ratio greater than 20:1 at initiation of therapy, vancomycin doses greater than 4 grams in 24 hours, and higher serum vancomycin trough levels have all been found to have an association with the development of AKI while receiving vancomycin. ${ }^{7,8}$ It is also thought that more aggressive treatment goal troughs of greater than $15 \mathrm{mg} / \mathrm{dL}$, supported by treatment guidelines, may contribute to the rate of vancomycin-associated AKI as well. ${ }^{1}$ The incidence of AKI has been reported as high as $43 \%$ in critically-ill patients being treated with vancomycin. ${ }^{9}$

Studies have been conducted, in pediatric and adult populations, evaluating risk-factors of vancomycin-associated AKI.,8,10-13 However, pediatric and adult populations differ in numerous ways and the development from childhood to adulthood differs among individuals. ${ }^{14}$ Variations in all aspects of pharmacokinetics and pharmacodynamics are present between these two populations. ${ }^{15}$ There has yet to be any studies assessing risk factors of vancomycinassociated AKI, caused by vancomycin, specifically in the adolescent and young adult population. Therefore, in this study, we aimed to determine the incidence of vancomycin- 
associated AKI in critically ill adolescent and young adult-patients and evaluate the risk factors associated with development of this adverse event in patients on vancomycin therapy.

\section{Methods}

This study was a multi-center, retrospective chart review conducted at Indiana University (IU) Health's downtown Academic Health Center (AHC) facilities located in Indianapolis, IN. This includes IU Health Methodist Hospital (625 bed adult hospital), IU Health University Hospital (339 bed adult hospital), and Riley Hospital for Children (255 bed pediatric hospital). At these institutions vancomycin is dosed by clinical pharmacists through a dosing consult protocol. This protocol does not lay out how vancomycin should be dosed or adjusted but allows the clinical pharmacist to dose and adjust the vancomycin based on their clinical judgement.

After receiving approval by the Institutional Review Board, patients aged 15 - 25 years who received at least 48 hours of vancomycin while in the intensive care unit from July 1, 2014 to June 30, 2016 were screened for inclusion to the study. Patients were included if they had a serum creatinine $(\mathrm{Scr})$ value within the 48 hours prior to vancomycin initiation and at least one serum vancomycin trough level.

Patients were excluded from analysis if they had a baseline $\mathrm{Scr}>1.5 \mathrm{mg} / \mathrm{dL}, \mathrm{BMI}$ of $\leq 15$ $\mathrm{kg} / \mathrm{m}^{2}$ or $\geq 40 \mathrm{~kg} / \mathrm{m}^{2}$, were an amputee, pregnant, had baseline kidney disease, a cystic fibrosis (CF) diagnosis, or were on extracorporeal membrane oxygenation (ECMO) or renal replacement therapy.

Acute kidney injury in this population was defined as an increase in serum creatinine by $0.5 \mathrm{mg} / \mathrm{dL}$ or $50 \%$ from baseline for two consecutive days. Upon identification of AKI incidence 
rate, patients were compared in two separate arms: 1) patients who developed AKI 2) patients who did not develop AKI. Data evaluated included: daily vancomycin dose, vancomycin trough levels, duration of vancomycin therapy, concurrent vasopressors, concurrent nephrotoxic medications (acyclovir, aminoglycosides, amphotericin B, calcineurin inhibitors, iodinated contrast agents, non-steroidal anti-inflammatory drugs [NSAID], piperacillin/tazobactam), procedures during therapy, and length of intensive care unit (ICU) stay.

Presence of vancomycin-associated AKI was analyzed with an incidence rate. Descriptive statistics were utilized for subgroup analysis and identification of risk factors for the occurrence of AKI while on vancomycin. Categorical data were evaluated using Chi-squared and Fisher's exact tests and continuous data were evaluated with the Wilcoxon Mann-Whitney U test. A pvalue $<0.05$ was considered as statistically significant. Analysis was performed in SPSS statistical software (IBM Corp., Armonk, N.Y., USA).

\section{Results}

A total of 418 vancomycin treatment courses were administered from July 1, 2014 to June 30, 2016 with 119 patients meeting initial inclusion. From these 119 patients, 69 patients were excluded due to a cystic fibrosis diagnosis, baseline kidney damage, BMI greater than 40, pregnancy, or ECMO cannulation; this resulted in a final study population of fifty patients. Twenty patients (40\%) developed AKI. There were no differences in baseline demographics as seen in Table 1.

There were no significant differences in vancomycin daily dose or duration of vancomycin therapy. However, median highest vancomycin trough level was $31.15 \mathrm{mcg} / \mathrm{mL}$ (11.28-46.68) in the AKI group compared to $12.5 \mathrm{mcg} / \mathrm{mL}(8.73-16.7)$ in the no AKI group 
$(p=0.006)$. Percent of patients that underwent procedure during vancomycin therapy were significantly higher in the AKI group (35\%) than the no AKI group (6.7\%) $(p=0.021)$. The procedures reported in these patients were orthopedic surgery $(n=3)$, chest tube insertion $(n=2)$, bone marrow aspiration $(n=2)$, intestine transplant, and ventriculoperitoneal shunt placement $(n=1)$. Table 2 summarizes the differences of factors between the two groups

Patients who developed AKI had higher numbers of concurrent nephrotoxic medications than those who did not develop AKI $(p=0.012)$. Figure 1 demonstrates that the presence of at least one concurrent nephrotoxic medication was also significantly higher in the AKI group with $95 \%$ compared to $60 \%$ in the no AKI group ( $p=0.012)$. The most common concurrent nephrotoxic medication was piperacillin/tazobactam. Of patients who received at least one nephrotoxic medication, $65 \%$ received piperacillin/tazobactam in the no AKI group and $43.3 \%$ in the AKI group. Like the concurrent nephrotoxic medications, Figure $\mathbf{2}$ shows that concurrent vasopressor usage was higher in the AKI group compared to the no AKI group at 55\% and $23 \%$ $(p=0.029)$.

\section{Discussion}

To date, this is the first study that has evaluated the incidence of vancomycin-associated AKI in a focused population of critically ill adolescent and young adult patients. Here we report a $40 \%$ incidence of AKI in critically-ill adolescent and young adult patients. This is consistent with previously published studies evaluating vancomycin-associated AKI in critically-ill adult and pediatric patients that have reported a variable incidence from $5 \%$ to $43 \% .{ }^{9}$ Factors such as high vancomycin doses, high vancomycin serum trough concentrations, and concurrent nephrotoxic medications have been identified as predictors of vancomycin-associated AKI in critically ill 
children. These factors, in addition to longer vancomycin days of therapy, obesity, and higher APACHE II scores, were associated with vancomycin-associated AKI in critically ill adults. In our study, we did not find a significant difference in vancomycin daily dose or duration of vancomycin therapy duration in patients that developed AKI compared to those that did not develop AKI. In fact, total daily dose of vancomycin was actually lower in patients who developed AKI. Patients that developed AKI did have a higher maximum vancomycin trough concentration in our study. Several studies have reported a significant association of high vancomycin serum trough concentrations with nephrotoxicity $7,16,17$. Additionally, a report by Horey and colleagues identified a linear relationship between higher incidence of AKI and graded trough concentrations. ${ }^{18}$ Like the majority of previous evaluations of risk factors in vancomycin-associated AKI, this study identified a significant association with higher vancomycin trough concentrations and incidence of AKI. This factor is difficult to interpret however, as it is not clear whether the kidney injury is a result of the higher trough level or if the level is a result of the reduction in clearance due to the kidney injury. Aside from routine, steady-state levels, random vancomycin trough levels are often checked when there is a change in the patient's clinical status or with a suspected change in clearance/renal function. For the patients with a suspected decrease in renal function, the injury may have already occurred, resulting in the elevated level.

Similar to preceding studies reporting incidence of vancomycin AKI, patients in our study that developed AKI had higher numbers of concurrent nephrotoxic medications than those who did not develop AKI. In a study evaluating acute kidney injury during vancomycin therapy in critically ill children, Totapally et al reported an association between the administration of 
concurrent nephrotoxic medications and the incidence of acute kidney injury. ${ }^{8} \operatorname{In} 2015$, Knoderer and colleagues found that the risk of AKI was higher in pediatric patients with concurrent use of vancomycin with acyclovir, amphotericin B, or piperacillin-tazobactam. ${ }^{19}$ Adult studies have identified vancomycin in addition to piperacillin-tazobactam or aminoglycosides has also resulted in increased rates of AKI. ${ }^{20,21}$

We have shown that the presence of concurrent vasopressors was associated with increased AKI. Jeffres et al. also found this association in adult patients who were being treated with vancomycin for MRSA pneumonia. ${ }^{22}$ Vasopressors and acute kidney injury have been a topic of discussion for many years. ${ }^{23}$ Increasing severity of hypotension has been associated with increased incidence of acute kidney injury in critically-ill patients. ${ }^{24}$ Patients requiring more vasopressors likely indicate a higher severity of hypotension. Similar to concurrent nephrotoxic medications, there were a significantly higher number of patients on at least one concurrent vasopressor who developed AKI in the critically-ill adolescent and young adult population in this study.

In our study, we have shown that patients who underwent a procedure were more likely to experience AKI. Surgical procedures can cause large shifts in fluid status and an increase in insensible losses. ${ }^{25}$ This can result in pharmacokinetic changes with vancomycin. These patients may require earlier and more frequent vancomycin trough level follow-up to help prevent this further insult to the kidneys.

We recognize that this study had some limitations. First, due to the retrospective study design and missing data, it was not feasible to calculate a severity-of-illness score. While there were no differences in baseline characteristics between groups, the lack of this score makes it 
difficult to understand how similar the groups were as it relates to acute kidney injury and vancomycin compared to AKI from critical illness alone. Second, the small sample size and study design prevented us from detecting any true causative factors. Lastly, we did not evaluate any long-term morbidity or mortality data in this study, thus the permanent effects cannot be determined. A prospective study would be required to confirm these results.

\section{Conclusion}

Vancomycin-associated AKI occurred in $40 \%$ of critically-ill adolescent and young adult patients. These patients may be more likely to develop vancomycin-associated AKI in the presence of high vancomycin trough levels, concurrent nephrotoxic agents, and concurrent vasopressor therapy. Patients undergoing a procedure may also be at an increased risk developing vancomycin-associated AKI and should be monitored with early trough levels following surgery. 


\section{References}

1. Rybak M, Lomaestro B, Rotschafer JC, et al. Therapeutic monitoring of vancomycin in adult patients: A consensus review of the American Society of Health-System Pharmacists, the Infectious Diseases Society of America, and the Society of Infectious Diseases Pharmacists. Am J Heal Pharm. 2009;66(1):82-98.

2. Liu C, Bayer A, Cosgrove SE, et al. Clinical practice guidelines by the Infectious Diseases Society of America for the treatment of methicillin-resistant Staphylococcus aureus infections in adults and children. Clin Infect Dis. 2011;52(3).

3. Glover ML, Cole E, Wolfsdorf J. Vancomycin dosage requirements among pediatric intensive care unit patients with normal renal function. J Crit Care. 2000;15(1):1-4.

4. Levine DP. Vancomycin : A History. Clin Infect Dis. 2006;42(Suppl 1):S5-12.

5. Öktem F, Arslan MK, Ozguner F, et al. In vivo evidences suggesting the role of oxidative stress in pathogenesis of vancomycin-induced nephrotoxicity: Protection by erdosteine. Toxicology. 2005;215(3):227-233.

6. Dieterich C, Puey A, Lyn S, et al. Gene expression analysis reveals new possible mechanisms of vancomycin-induced nephrotoxicity and identifies gene markers candidates. Toxicol Sci. 2009;107(1):258-269.

7. Hanrahan TP, Kotapati C, Roberts MJ, et al. Factors associated with vancomycin nephrotoxicity in the critically ill. Anaesth Intensive Care. 2015;43(5):594-599.

8. Totapally BR, Machado J, Lee H, Paredes A, Raszynski A. Acute kidney injury during Vancomycin Therapy in critically ill children. Pharmacotherapy. 2013;33(6):598-602. 
9. Van Hal SJ, Paterson DL, Lodise TP. Systematic review and meta-analysis of vancomycininduced nephrotoxicity associated with dosing schedules that maintain troughs between 15 and 20 milligrams per liter. Antimicrob Agents Chemother. 2013;57(2):734-744.

10. Moffett BS, Hilvers PS, Dinh K, Arikan AA, Checchia P, Bronicki R. Vancomycin-associated acute kidney injury in pediatric cardiac intensive care patients. Congenit Heart Dis. 2014;10(1):E6-E10.

11. Hanrahan TP, Harlow G, Hutchinson J, et al. Vancomycin-Associated Nephrotoxicity in the Critically III: A Retrospective Multivariate Regression Analysis. Crit Care Med. 2014;(c):1-10.

12. McKamy S, Hernandez E, Jahng M, Moriwaki T, Deveikis A, Le J. Incidence and risk factors influencing the development of vancomycin nephrotoxicity in children. J Pediatr. $2011 ; 158(3): 422-426$.

13. Bamgbola O. Review of vancomycin-induced renal toxicity: an update. Ther Adv Endocrinol Metab. 2016;7(3):136-147.

14. Lanke S, Yu T, Rower JE, Balch AH, Korgenski EK, Sherwin CM. AUC-Guided Vancomycin Dosing in Adolescent Patients With Suspected Sepsis. J Clin Pharmacol. 2016;57(1):77-

84.

15. Kearns GL, Abdel-Rahman SM, Alander SW, Blowey DL, Leeder JS, Kauffman RE. Developmental pharmacology--drug disposition, action, and therapy in infants and children. N Engl J Med. 2003;349(12):1157-1167. 
16. Cano EL, Haque NZ, Welch VL, et al. Incidence of Nephrotoxicity and Association With Vancomycin Use in Intensive Care Unit Patients With Pneumonia: Retrospective Analysis of the IMPACT-HAP Database. Clin Ther. 2012;34(1):149-157.

17. Elyasi S, Khalili H, Dashti-Khavidaki S, Mohammadpour A. Vancomycin-induced nephrotoxicity: Mechanism, incidence, risk factors and special populations. A literature review. Eur J Clin Pharmacol. 2012;68(9):1243-1255.

18. Horey A, Mergenhagen K a, Mattappallil A. The Relationship of nephrotoxicity to vancomycin trough serum concentrations in a veteran's population: a retrospective analysis. Ann Pharmacother. 2012;46(11):1477-1483.

19. Knoderer CA, Gritzman AL, Nichols KR, Wilson AC. Late-occurring vancomycin-associated acute kidney injury in children receiving prolonged therapy. Ann Pharmacother. 2015;49(10):1113-1119.

20. Burgess LD, Drew RH. Comparison of the incidence of vancomycin-induced nephrotoxicity in hospitalized patients with and without concomitant piperacillintazobactam. Pharmacotherapy. 2014;34(7):670-676.

21. Rybak MJ, Albrecht LM, Boike SC, Chandrasekar PH. Nephrotoxicity of vancomycin, alone and with an aminoglycoside. J Antimicrob Chemother. 1990;25(4):679-687.

22. Jeffres MN, Isakow W, Doherty JA, Micek ST, Kollef MH. A retrospective analysis of possible renal toxicity associated with vancomycin in patients with health careassociated methicillin-resistant Staphylococcus aureus pneumonia. Clin Ther. 2007;29(6):1107-1115. 
23. Bellomo R, Wan L, May C. Vasoactive drugs and acute kidney injury. Crit Care Med. 2008;36(Suppl):S179-S186.

24. Lehman L-W, Saeed M, Moody G, Mark R. Hypotension as a Risk Factor for Acute Kidney Injury in ICU Patients. Comput Cardiol (2010). 2010;37(2010):1095-1098.

25. Strunden MS, Heckel K, Goetz AE, Reuter DA. Perioperative fluid and volume management: physiological basis, tools and strategies. Ann Intensive Care. 2011;1:2. 
Table 1: Summary of demographic data for patients included in final analysis

\begin{tabular}{|l|c|c|}
\hline Characteristic & No AKI (n=30) & AKI (n=20) \\
\hline $\begin{array}{l}\text { Age } \\
\text { a }\end{array}$ & $20.5(19-22.25)$ & $18.5(17-21)$ \\
\hline $\begin{array}{c}\text { Mender } \\
\text { Male }\end{array}{ }^{a}$ & $19(63.3 \%)$ & $12(60 \%)$ \\
\hline Weight $^{a}$ & $57.8(52.63-84.28)$ & $73.75(54.55-94.05)$ \\
\hline BMI & $21.9(18.56-27.43)$ & $27.2(19.84-33.46)$ \\
\hline Baseline Scr & $0.58(0.46-0.73)$ & $0.63(0.37-0.78)$ \\
\hline
\end{tabular}

aMedian (IQR)

No significant differences were found between demographic data of the two groups 
Table 2: Differences in evaluated factors between study groups

\begin{tabular}{|c|c|c|c|}
\hline Characteristic & No AKI $(n=30)$ & AKI $(n=20)$ & P value \\
\hline 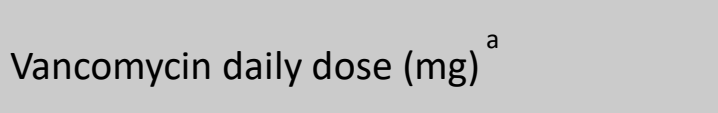 & $\begin{array}{c}3000 \\
(2187.5-3750)\end{array}$ & $\begin{array}{c}2570 \\
(2062.5-3637.5)\end{array}$ & 0.921 \\
\hline Vancomycin daily dose $(\mathrm{mg} / \mathrm{kg})^{\mathrm{a}, \mathrm{b}}$ & $\begin{array}{c}43.82 \\
(36.99-53.72)\end{array}$ & $\begin{array}{c}37.78 \\
(28.88-51.04)\end{array}$ & 0.198 \\
\hline Duration of therapy (days) ${ }^{a}$ & $5.5(4-8)$ & $6.5(4-7)$ & 0.540 \\
\hline Highest vancomycin trough $(\mathrm{mg} / \mathrm{dL})^{a}$ & $\begin{array}{c}12.5 \\
(8.73-16.7)\end{array}$ & $\begin{array}{c}31.15 \\
(11.28-46.68)\end{array}$ & 0.006 \\
\hline Concurrent vasopressors (n) ${ }^{a}$ & $0(0-0.25)$ & $1(0-1)$ & 0.029 \\
\hline Concurrent nephrotoxic medications $(n)^{a}$ & $1(0-2)$ & $2(1-3)$ & 0.012 \\
\hline Procedure & $2(6.7 \%)$ & $7(35 \%)$ & 0.021 \\
\hline Length of ICU stay (days) ${ }^{a}$ & $7.5(4-14.25)$ & $9.5(7-24)$ & 0.081 \\
\hline \multicolumn{4}{|l|}{ aMedian (IQR) } \\
\hline${ }^{b}$ Actual body weight & & & \\
\hline
\end{tabular}




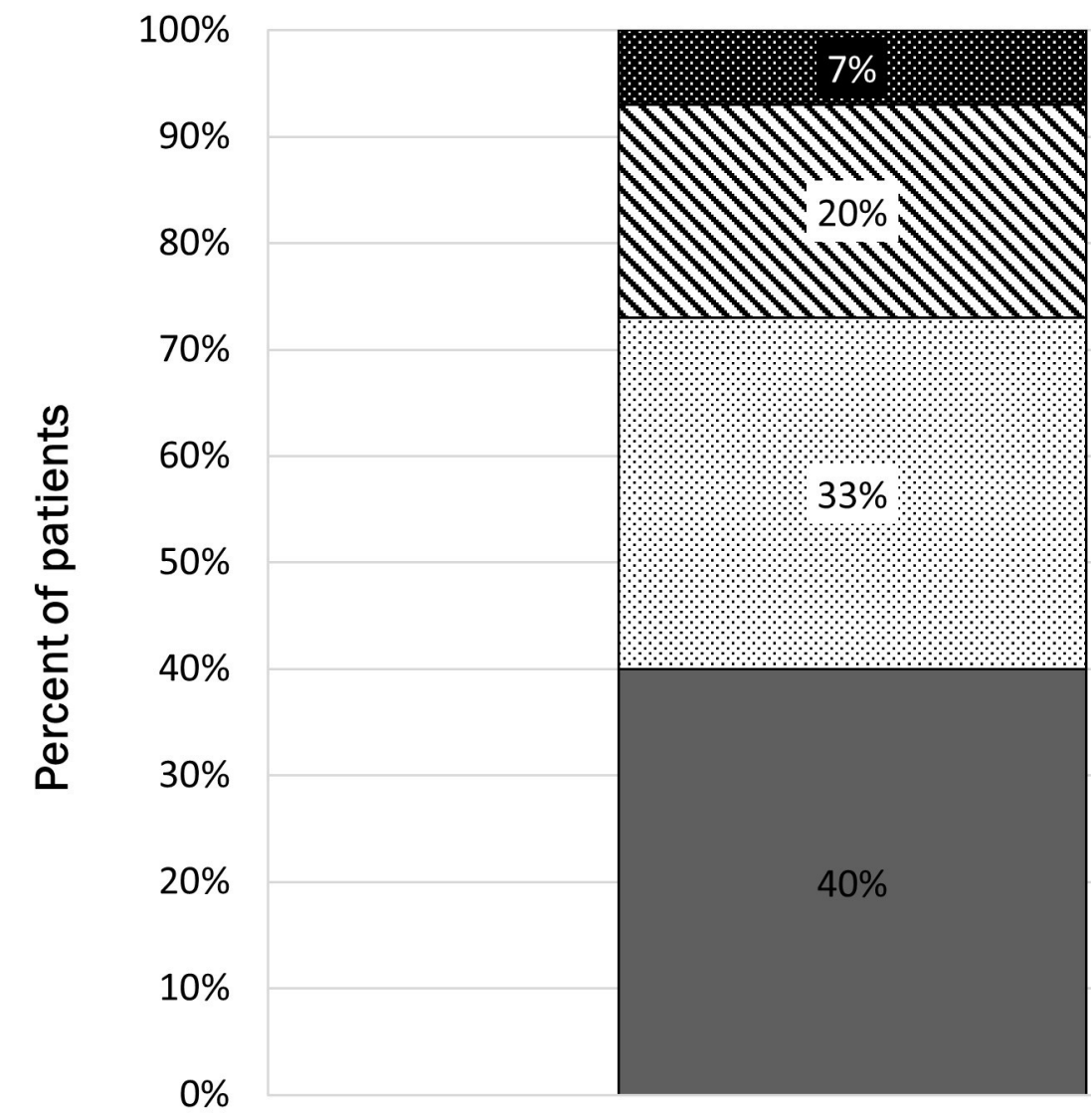

No AKI

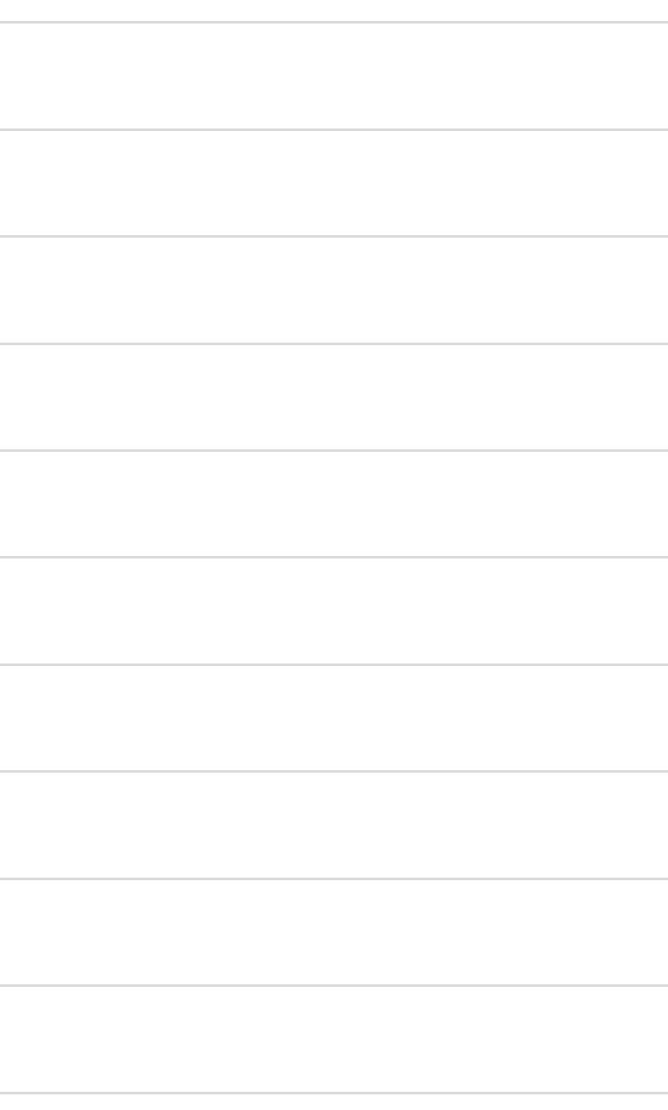

$\square 0 \quad 81$ \$2 83

Number of concurrent nephrotoxic medications

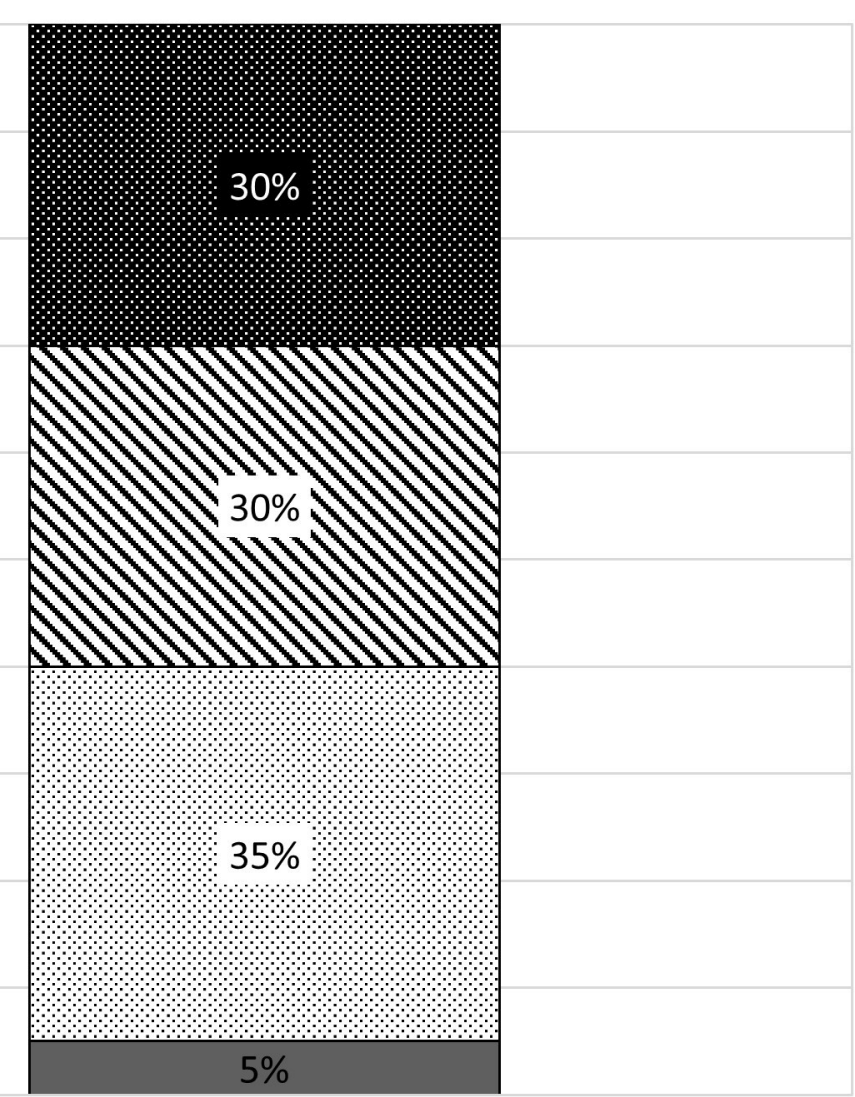

AKI 


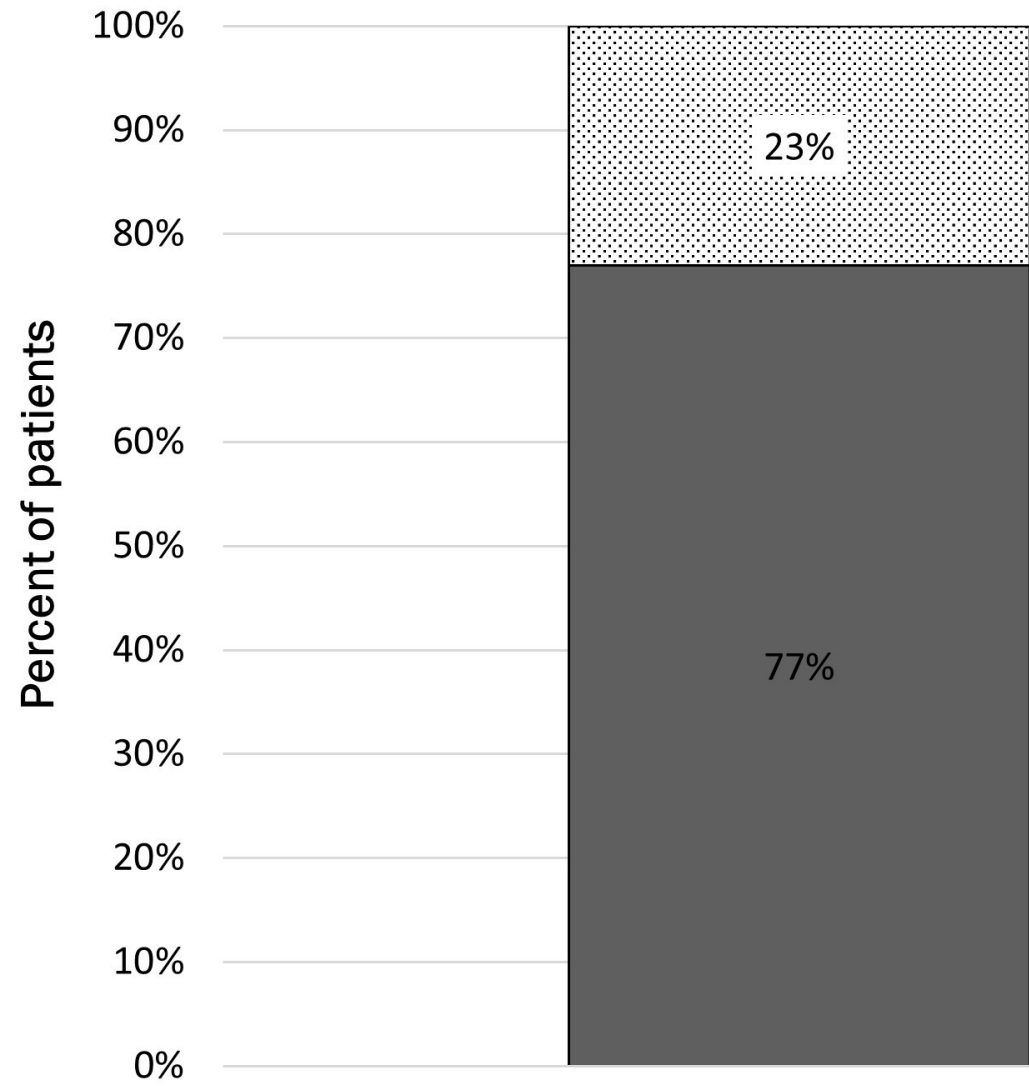

No AKI

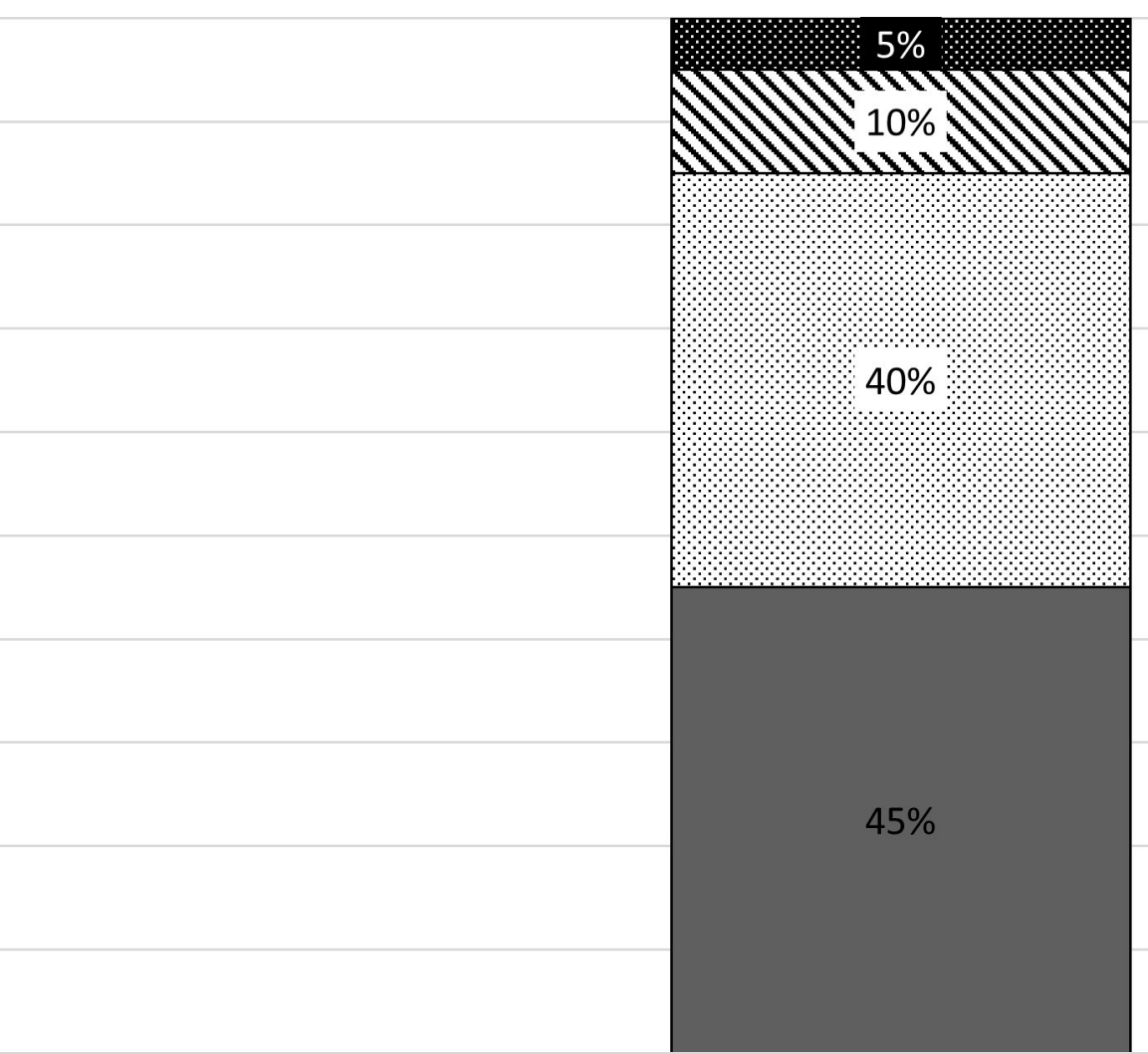

AKI

Number of concurrent vasopressors 